\title{
Insular Cortex Activity and the Evocation of Laughter
}

\author{
Elise Wattendorf, ${ }^{1 *}$ Birgit Westermann, ${ }^{2}$ Martin Lotze, ${ }^{3}$ Klaus Fiedler, ${ }^{1}$ and Marco R. Celio ${ }^{1}$ \\ ${ }^{1}$ Anatomy and Program in Neuroscience, Department of Medicine, University of Fribourg, $\mathrm{CH}-1700$ Fribourg, Switzerland \\ ${ }^{2}$ Department of Neurosurgery, University Hospital, University of Basel, $\mathrm{CH}-4031$ Basel, Switzerland \\ ${ }^{3}$ Functional Imaging Unit, Diagnostic Radiology, University Hospital, University of Greifswald, D-17489 Greifswald, Germany
}

\section{ABSTRACT}

The insular cortex is fundamentally involved in the processing of interoceptive information. It has been postulated that the integrative monitoring of the bodily responses to environmental stimuli is crucial for the recognition and experience of emotions. Because emotional arousal is known to be closely coupled to functions of the anterior insula, we suspected laughter to be associated primarily with neuronal activity in this region. An anatomically constrained re-analysis of our imaging data pertaining to ticklish laughter, to inhibited
\end{abstract}

ticklish laughter, and to voluntary laughter revealed regional differences in the levels of neuronal activity in the posterior and mid-/anterior portions of the insula. Ticklish laughter was associated specifically with right ventral anterior insular activity, which was not detected under the other two conditions. Hence, apparently, only laughter that is evoked as an emotional response bears the signature of autonomic arousal in the insular cortex.

INDEXING TERMS: insular cortex; anterior insula; laughter; emotional arousal; fMRI; autonomic; positive emotion; tickle

On the basis of pathological findings and of imaging data from healthy individuals, information has been gathered regarding the brain regions that are implicated in the expression of laughter (Wild et al., 2003; Lauterbach et al., 2013), which confirms Wilson's concept (1924) that, during this form of vocalization, a voluntarily controlled system inhibits an emotionally mediated one. The brainstem is critically involved; volitional motor signals are delivered to the ventral tegmentum via corticopontine projections, whereas the periaqueductal gray (PAG), the dorsal tegmentum, and the brainstem nuclei, which are essential for the emotional motor system, receive descending inputs from the orbitofrontal and insular cortices, the amygdala, and the hypothalamus. Although evidence favoring an involvement of the PAG and the hypothalamus in the generation of motor patterns of laughter is convincing (Altafullah et al., 1988; Davis et al., 1996; Dujardin and Jürgens, 2006; Jürgens, 2009), that implicating the amygdala and the orbitofrontal and insular cortices in this process is more ambiguous. Watson and his colleagues (2007) have proposed that the anterior insula responds to the dynamics of the emotional changes that accompany the recognition and the appraisal of a humorous experience. Indeed, an association has been reported between pathological laughter and a stroke-impacted insula (Carel et al.,
1997; Osseby et al., 1999), although the resolution of the images in the afflicted patients did not suffice to pinpoint the implicated subregions. Hitherto, imaging studies pertaining to the processing of humor or tickling have not yielded conclusive data, insofar as the activation in the insular cortex has not been described in detail and the effective vocal response has not been recorded (Carlsson et al., 2000; Wild et al., 2006; Schwartz et al., 2008). Hence, the present Review refers mainly to our own fMRI findings on the expression of ticklish laughter and on the inhibition of ticklish laughter and of voluntary laughter in healthy individuals (Wattendorf et al., 2013). The aim of this re-analysis of our data was to relate neuronal activity in subregions of the insular cortex to specific functions. The insula is a cortical lobe that lies hidden within the depth of the lateral sulcus. It can be coarsely subdivided into two regions: a mid-/anterior portion (gyri breves insulae) 
and a posterior one (gyri longi insulae). In the past, circuitries and functional aspects of the insular cortex have been studied not only in rodents but also in primates, including humans (Mufson and Mesulam, 1982; Mesulam and Mufson, 1982a,b; Allen et al., 1991; Dupont et al., 2003). The posterior insula receives projections from all sensory systems that convey information pertaining to the physiological condition of bodily tissues. In progressive re-representations, the stimuli are then processed bilaterally in more anterior parts of the insula, permitting a refinement of the primary interoceptive information (Craig, 2003). For example, midportions of the insula are functionally interconnected with an array of structures, such as the nucleus accumbens, the amygdala, the hypothalamus, and the ventromedial prefrontal cortex, and diverse sensory modalities, which facilitate the integration of concomitant sensory, motor, and limbic-related activities. This integrative process underlies the emergence of bodily feelings. The anterior portions of the insula, together with their connections to the prefrontal, orbitofrontal, and anterior cingulate cortex, embrace central-network activity patterns, which afford the basis for emotional and subjective feelings. The latter are, in turn, associated with the bodily needs for homeostasis (Craig, 2009). Experiments with rats have revealed the autonomic efferent connections from the anterior insula to be conveyed primarily via a relay system in the lateral hypothalamic area (Cechetto and Chen, 1990; Yasui et al., 1991). Imaging data have disclosed an involvement of the anterior insula in positive and negative emotional experiences (Damasio et al., 2000; Anders et al., 2004). Not surprisingly, this region is also activated during a humorous experience (Moran et al., 2004; Watson et al., 2007) and during the typical vocal expression of a positive emotion, namely, laughter (Wattendorf et al., 2013). Here, the anterior insula is believed to respond to the dynamics of the emotional changes that accompany the recognition and appraisal of a humorous experience (Watson et al., 2007).

In humans, laughter can be evoked spontaneously, as an emotional response, or voluntarily, for a social purpose, and it can frequently even be suppressed. Intense amusement or true laughter is accompanied by immediate sympathetic arousal, whereas the active inhibition of an emotional outburst suppresses this response (Giuliani et al., 2008; Lackner et al., 2014). Of particular interest is the concomitant emotional processing of information in the insular cortex during the experience of pleasurable feelings with and without laughter and laughter with and without the corresponding feelings. In a previous report (Wattendorf et al., 2013), we mapped the fMRI signals in the insula with- out specifically alluding to their subregional distribution, and the activity levels under the conditions of ticklish laughter (T), of inhibited ticklish laughter (I), and of voluntary laughter (V) were not compared. In the present re-analysis of these data, the aim was to distinguish the regional patterns of activation in the insula, particularly in its anterior portion, under these three conditions and to evaluate our findings in light of the data from other imaging studies pertaining to laughter.

\section{MATERIALS AND METHODS}

Our present analysis is based on data that have been documented in a previous publication (Wattendorf et al., 2013). In this former investigation, the fMRI-signals that were generated in 18 individuals under the three conditions of $\mathrm{T}, \mathrm{I}$, and $\mathrm{V}$ were assessed. During the scanning session, a friend (for 11 individuals) or the partner (for seven individuals) of the participant stood in the scanning room and tickled or touched the right foot according to the particular stimulus condition. Each of the three tested conditions was indicated by a specific visual stimulus, consisting of a specific "smiley face" which was projected on separate screens for the tickler and the tickled person. Spontaneous laughter was evoked by tickling the sole of the right foot. Individuals were permitted to express the response during the condition $\mathrm{T}$ but were requested to suppress it during the condition I. Individuals were required to produce laughter on demand during the condition V. Each of the three conditions was randomly presented 20 times, each lasting 6.2 seconds, and alternated with the presentation of a cross-signaled period of rest (11 seconds). Laughter events were recorded during the entire MRI scanning procedure. It is worthy of mention here that technical and methodological difficulties are frequently encountered when testing a paradigm that is associated with physical movements. To prevent head movements and ensuing susceptibility artifacts in the fMRI signal, the participants held a wooden barbecue stick between their teeth during the course of the experiment, which did not interfere with laughter. Nevertheless, nine of the originally 27 participants in our study had to be excluded from the evaluation process because the amplitude of their head movements consistently exceeded voxel size during the scanning procedure.

Imaging was performed on a 3-T Scanner (Verio; Siemens, Erlangen, Germany) with a 12-channel head coil. Functional images were obtained with a $\mathrm{T} 2^{*}$-weighted echo planar imaging (EPI) sequence (repetition time 2.2 seconds, echo time $30 \mathrm{msec}$, flip angle $90^{\circ}$, resolution 3.0 $\times 3.0 \times 3.0 \mathrm{~mm}$, with a $1-\mathrm{mm}$ gap). To minimize susceptibility artifacts, images were additionally tilted by $30^{\circ}$ 


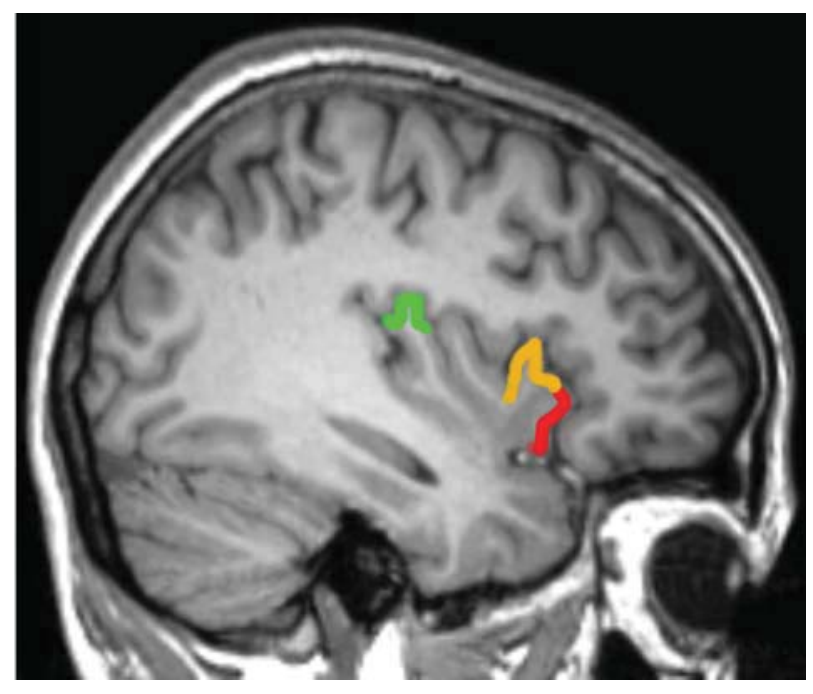

Figure 1. Lateral view on the left insular cortex. The primary interoceptive cortex in the posterior and anterior areas of the dorsal fundus of the insula (idfp and idfa) are marked in green, and the dorsal and ventral portions of the anterior insular cortex (daic, vaic) are labeled in yellow and red, respectively. relative to the anterior/posterior commissure and recorded in interleaved mode. Data were analyzed in SPM5 software (Wellcome Department of Cognitive Neuroscience, London, England). To correct for movement artifacts, each individual scan was aligned to the first scan. During this procedure, small variations are weighted highly, and strong variance in signal strength is weighted less by means of a within-modality rigid registration. Movement parameters estimated during the realignment procedure were introduced as covariates into the model to control for variance resulting from head displacements in correlative mode. An event-related analysis was used to identify neural activity following stimulation for $\mathrm{T}$, I, and $\checkmark$ separately. Additional technical details, as well as the evaluation protocol, have been fully described in a previous publication (Wattendorf et al., 2013).

In the present study a region-of-interest (ROI) analysis covering the insular cortex of both hemispheres was conducted with a view to relating regional activation patterns to spontaneous or voluntary laughter and the suppression of the affective vocal response to tickling. The insular cortex was subdivided according to data

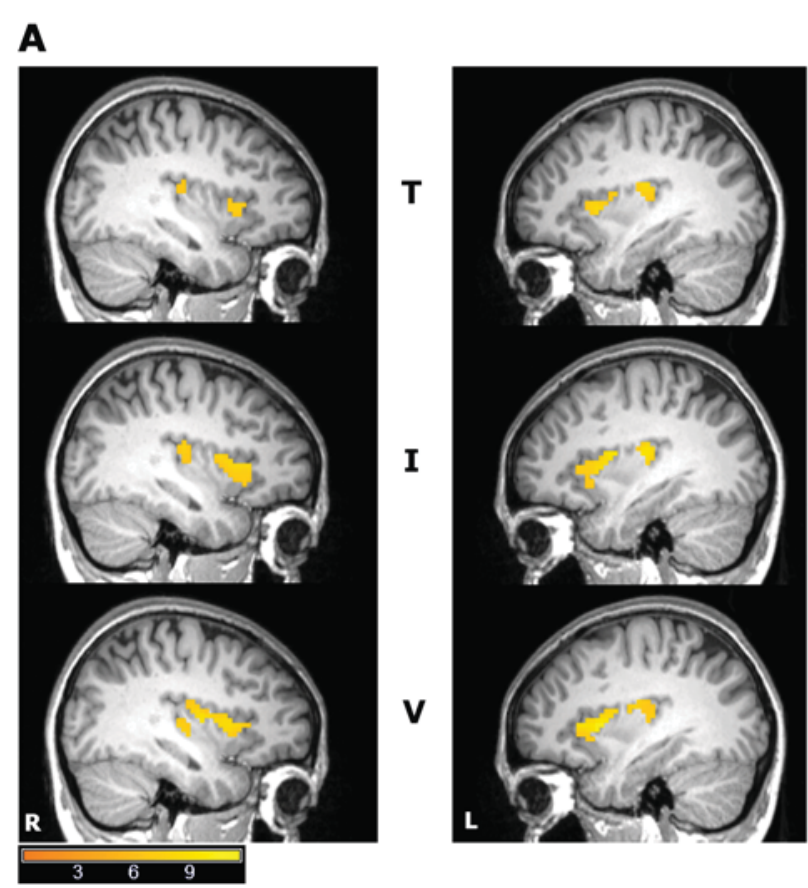

B
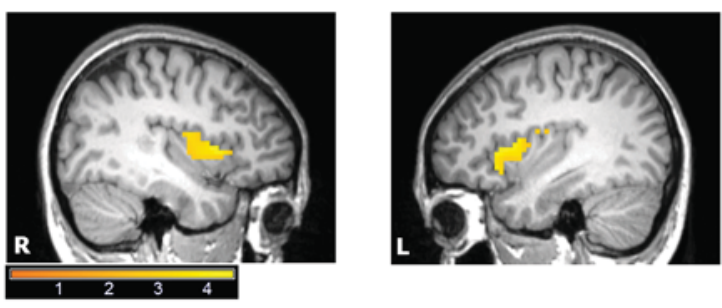

C

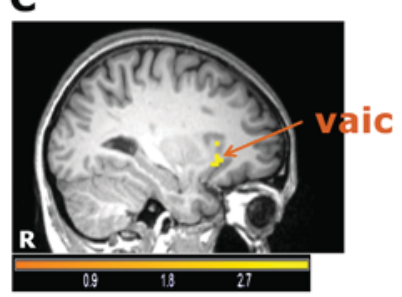

Figure 2. Insular activity during ticklish laughter (T), inhibited ticklish laughter (I), and voluntary laughter (V). A: Virtual sagittal slices through a human brain, revealing heightened levels of activity (main effect; $P<0.001 \mathrm{FWE}$ ) in the insula (ROI analysis) during T, I, and V. $R$, right hemisphere; $L$, left hemisphere. Under all three conditions, the slices were aligned according the activation maximum during $T$ in the posterior insula of each hemisphere separately. B: Virtual sagittal slices revealing significant $\mathrm{V}>\mathrm{T}$ contrast differences $(P<0.005$, uncorrected) in the insula (ROI analysis). C: Virtual sagittal slice through the right hemisphere (at $\mathrm{x}=30)$, depicting the activation maximum in the insular cortex during $T$ and the corresponding laughter events (correlation analysis at $P<0.005$, uncorrected; ROI analysis). VAIC, ventral anterior insular cortex. In the correlation, $r=0.65, \mathrm{R}^{2}=0.43, P=0.003$. 
TABLE 1.

Insular Activity During Ticklish Laughter (T), Inhibited Ticklish Laughter (I), and Voluntary Laughter (V) ${ }^{1}$

\begin{tabular}{|c|c|c|c|c|c|c|c|c|}
\hline & \multicolumn{4}{|c|}{ Posterior insula } & \multicolumn{4}{|c|}{ Mid/anterior insula } \\
\hline & Side & Number of voxels & MNI coordinates & Z-score & Side & Number of voxels & MNI coordinates & Z-score \\
\hline \multicolumn{9}{|c|}{ Main effect } \\
\hline \multirow[t]{2}{*}{$\mathrm{T}$} & L & 40 & $-33-21 \quad 18$ & 5.24 & L & 68 & -36123 & 5.34 \\
\hline & $\mathrm{R}$ & 6 & $36-18 \quad 15$ & 4.64 & $\mathrm{R}$ & 45 & 39180 & 5.13 \\
\hline \multirow[t]{2}{*}{1} & L & 42 & $-36-186$ & 5.55 & L & 128 & $\begin{array}{lll}-39 & 9 & 0\end{array}$ & 5.59 \\
\hline & $\mathrm{R}$ & 18 & $36-18 \quad 15$ & 5.05 & $\mathrm{R}$ & 95 & 39123 & 5.37 \\
\hline \multirow[t]{2}{*}{ V } & L & $A+P 238$ & $-33-1215$ & 5.10 & L & $A+P 238$ & $\begin{array}{lll}-36 & 9 & 0\end{array}$ & 6.04 \\
\hline & $\mathrm{R}$ & $A+P 189$ & $36-66$ & 5.50 & $\mathrm{R}$ & $A+P 189$ & 39123 & 5.49 \\
\hline \multicolumn{9}{|c|}{ Comparison } \\
\hline \multirow[t]{2}{*}{$V>T$} & & & - & & L & 28 & $-3618 \quad 3$ & 3.60 \\
\hline & & & - & & $\mathrm{R}$ & 25 & 39123 & 3.58 \\
\hline \multirow[t]{2}{*}{$T>1$} & L & 3 & $-33-2412$ & 3.15 & & & & \\
\hline & $\mathrm{R}$ & 18 & $36-216$ & 3.45 & & & & \\
\hline \multicolumn{9}{|c|}{ Correlation of laughter events } \\
\hline \multirow[t]{2}{*}{ With T } & L & 7 & $-42-90$ & 2.85 & L & 3 & $\begin{array}{lll}-33 & 15 & -18\end{array}$ & 2.71 \\
\hline & $\mathrm{R}$ & 1 & $39-30$ & 2.58 & $\mathrm{R}$ & 11 & $3021-6$ & 2.94 \\
\hline With I & & & - & & & & - & \\
\hline
\end{tabular}

${ }^{1}$ The atlas coordinates are indicated in MNI space and the peak activations as Z-scores. The signals in the reported regions exceeded the threshold level of $P<0.001$ (FWE) in the main effect, that of $P<0.001$ (uncorrected) in the comparison and that of $P<0.005$ (uncorrected) in the correlation.

that were gleaned from the OASIS-project (http://www. oasis-brains.org/) and that were furnished by Neuromorphometrics, Inc. (http://neuromorphometrics.com/). The anterior dorsal and anterior ventral insular fields were defined according to the labeling patterns reported by Wager and Barrett (2004). In their study, these regions of the human insular cortex and the overlying operculum were delineated by digitalized parcellation, for which the cytoarchitectural and fiber tract-tracing studies of Mesulam and Mufson served as a basis (Mufson and Mesulam, 1982; Mesulam and Mufson, 1982a,b). The cortical locations of dorsal and ventral anterior parts of the insula (daic, vaic) are depicted in Figure 1.

\section{RESULTS}

Imaging data pertaining to insular activity during ticklish laughter, inhibited ticklish laughter, and voluntary laughter are depicted visually and numerically in Figure 2 and Table 1, respectively. Bilateral activity in both the posterior and the mid-/anterior portions of the insular cortex was observed under all three conditions $(P<0.005$, FEW; Fig. 2A). However, during $\mathrm{T}$ and $\mathrm{I}$, the level of activity was higher in the left hemisphere than in the right one and thus contralateral to the side of the stimulation, both posteriorly and anteriorly, whereas during $\mathrm{V}$ the left-side prominence was apparent only anteriorly. During $\mathrm{T}$ and I, peak activity in the posterior insular cortex was bilaterally registered in the fundus of the superior limiting sulcus. The implicated region, namely, the posterior (idfp) and the anterior (idfa) areas of the dorsal fundus, correspond to the primary interoceptive granular cortex (Evrard et al., 2014). For a precise localization of these two brain regions see Figure 1. During $\mathrm{V}$, posterior insular activity was lower on the left than on the right, and it was not centered in the primary interoceptive cortex. Compared with the situation during $\mathrm{T}$, the activity levels in the dorsal mid-/anterior insular cortex were bilaterally higher during $\mathrm{V}(P<0.001$, uncorrected; Fig. 2B, Table 1). Differences in the levels of activity during $\mathrm{T}$ and I were observed only in the posterior portion of the insula (Table 1). The other paired comparisons revealed no significant differences in activitylevel bias. Laughter events that were evoked during $T$ correlated with the corresponding tickling situation $(P<0.005$, uncorrected); the association was most pronounced in the right ventral anterior and the left posterior portions of the insula (Fig. 2C, Table 1). During I, the degree of ticklish laughter had no bearing on the level of insular activity. A correlation between head movements and reported hemodynamic activity during $T$ was calculated for the right ventral anterior insula $(x=30, y=21$, $z=-6)$. Signal changes and realignment regressors for the translations in the z-direction (which, in each participant, corresponded to the most important ones) were computed by SPM5. Correlation of contrast estimates with interscan movement amplitudes (based on averages of the three scans following each stimulus onset) was not relevant $(r=0.13, P=0.92)$.

\section{DISCUSSION}

At a first sight, the levels of insular activity appeared to be comparably and bilaterally higher in the posterior 
and anterior portions of the cortex under each of the tested conditions, T, I, and V (Fig. 2A). However, a more meticulous analysis revealed the vocal response to the pleasurable experience during $T$ to differ significantly from that during either I or V. Most noteworthy is the higher activity level in the mid-/dorsal anterior portion during $\mathrm{V}$ than during $\mathrm{T}$ (Fig. 2B). Furthermore, laughter events that were evoked during $T$ were associated with BOLD activity in the right ventral anterior insula (Fig. 2C). Our findings are discussed below in the context of specific laughter-related functions and within the general framework of the functional insular subdivisioning that underlies the maintenance of organismic homeostasis.

\section{T involves the posterior and anterior insula}

In our investigation spontaneous laughter was evoked by tickling the right foot. As has been formerly reported for sensual touch (Olausson et al., 2002), the posterior insula was activated (Fig. 2A). The peak occurred in the superior limiting sulcus, a finding that accords with the known specialization of this area as a primary interoceptive cortical one (Evrard et al., 2014). Moreover, this activity was located contralaterally to the site of stimulation, as is usual for externally triggered responses in the primary somatosensory cortex. Our image analysis for $T$ revealed high signal intensities in the posterior portions of the insula and extensive bilateral activity in mid-/anterior ones. This latter finding is in accordance with data that have been documented in another publication related to tickling (Carlsson et al., 2000). The emotional feelings that are associated with tickling appear to involve the full insular activation sequence of interoceptive processing. On the other hand, in publications relating to the appreciation of humor, only the mid-/anterior portions of the insular cortex were activated (Moran et al., 2004; Watson et al., 2007). Hence, in these situations, the insular cortex appears to resort to other sources of emotional information.

\section{$T$ activates the ventral anterior insular cortex}

Our correlation analysis revealed the ventral anterior portion of the right insular cortex (Fig. 2C) to be specifically linked with the evocation of T. A functional distinction between the agranular ventral and the dysgranular dorsal portions of the anterior insula has been conceptualized (Wager and Barrett, 2004). The ventral anterior insular cortex, which is connected mainly to the orbitofrontal cortex, is believed to be important in motivational states that are related to core affect. For instance, previous imaging studies have revealed an experience of happiness, fear, disgust, sadness, or anger to be consistently associated with activity in the ventral anterior portions of the insula (Phillips et al., 1997; Damasio et al., 2000), pointing to the inherent motivational impact of these basic emotions. On the other hand, the dorsal anterior insula, with its proximity to the lateral prefrontal area, is believed to be crucial for the translation of motivational states into specific plans of action.

In an earlier report, a specialization of the cells within the ventral anterior portion of the insular cortex was discussed in the context of humor processing (Watson et al., 2007). Indeed, the portion of the human insula that adjoins the frontal operculum (the frontoinsular junction) is known to harbor von Economo (VEN) neurons (von Economo, 1926), which are phylogenetically of recent date. These neurons were noted for their distinctive morphology, first in humans and the great apes (von Economo, 1926; Nimchinsky et al., 1999; Allman et al., 2010) and later also in elephants (Hakeem 2009) and cetaceans (Butti et al., 2009). In these species, high numerical densities of VEN neurons are distributed selectively in specific regions of the frontal cortex, namely, in the anterior cingulate, the frontoinsular, and the frontopolar (dorsolateral prefrontal in hominids) cortices. Likewise, for other large vertebrates, VEN neurons have been reported to be broadly distributed throughout the cortex (Butti and Hof, 2010). It has been suggested that, phylogenetically, the confinement of VEN neurons to circumscribed regions of the brain has led to a progressive shaping of highly specialized neuronal pathways. In circuits involving cognitive, emotional, and social faculties, functions favoring social survival may have developed (Butti et al., 2013). Ancient, early branched organisms may have retained the original distribution, but any co-option to other functional pathways has not been ascertained. Investigations in humans implicate VEN neuron-containing cortical regions in the processing of salience, playing a role in the integration of external sensory information with internal emotional and bodily-state signals (Uddin, 2015). Moreover, VEN neurons are believed to be involved in the establishment of human awareness (Evrard et al., 2012) and have been implicated in the rapid, highly integrated representations of an emotional experience (Craig, 2009). An appreciation of humor is based on a reinterpretation of an emotional situation (Suls, 1972). The initially rapid intuitive interpretation of such a situation is followed by a slower cognitive updating process. During the recognition of humor, VEN neurons have been proposed to be "recalibrated according to the actual appraisal of the situation" (Watson et al., 2007), an event that may be relevant also during the processing of tickling: A situation that is intuitively appraised as being potentially threatening may be 
reappraised as harmless upon the actual contact with it (Ramachandran, 1998).

\section{During $\mathrm{T}$, activity in the ventral anterior insular cortex is lateralized to the right}

In general, emotional situations that are associated with a state of higher arousal, viz., those that prepare for a higher expenditure of energy, activate the right anterior insular cortex (for review see Craig, 2005). For instance, activity in the ventral portion of this region has been observed during the experience of a basic emotion such as happiness, fear, disgust, sadness, or anger, all of which can be accompanied by vocalization and/or intense bodily action (Phillips et al., 1997; Damasio et al., 2000). Studies that have focused on affective vocal behavior and/or the associated facial expressions have systematically revealed an asymmetric representation in the ventral anterior insular cortex, which was lateralized to the right when the activity of the vocal cords was targeted, such as during passive listening to laughter or crying (Sander and Scheich, 2005), or during overt singing (Riecker et al., 2000) but to the left part during situations in which facial expressions prevailed, such as during viewing or producing a smile (Hennenlotter et al., 2005) and when smiling at cartoons (Schwartz et al., 2008). On the basis of these findings, the ventral anterior patterns of neuronal activity are deemed to depend on the overriding type of behavioral response to the amusing situation; only during laughter is the pattern of activity believed to correspond to one of higher emotional arousal. Hence, our data strengthen the hypothesis that smiling and laughter not only represent different degrees of pleasant emotional expression but are fundamentally different from each other.

Asymmetric activity in the insula during laughter may also include a specific pattern of autonomic regulation. Isolated reports on stroke patients (Oppenheimer et al., 1992; Meyer et al., 2004), as well as imaging data (Lane 2009) and theoretical assumptions (Craig 2005), implicate the right anterior insular cortex in sympathetic efferent functions and the left portion in parasympathetic ones. As such, heightened right anterior ventral activity during $T$ would involve a (temporary) change in sympathetic control. However, in situations that elicited amusement, the sympathetic effects on skin conductance and heart rate did not yield convincing evidence in support of this tenet (Kreibig et al., 2013). On the other hand, in situations of intense amusement (Lackner et al., 2014) or positive emotional exaggeration (Demaree et al., 2004), a sympathetic effect could be clearly distinguished. It may be further speculated that the dynamics of autonomic signaling continue in other centers that are crit- ically involved in the control of emotional vocalization. For instance, concomitant activity has been recorded in the anterior insula and the hypothalamus during autonomic arousal related to positive emotions (Kuniecki et al., 2003) and, specifically, in the tuberolateral hypothalamic portion during the production of laughter (Wattendorf et al., 2013).

\section{Inhibited ticklish laughter}

During I, bilateral activity in the mid-/anterior insular cortex was at least equivalent to that evoked during $\mathrm{T}$, indicating that this situation is still associated with emotional feelings. In contrast, the urge to laugh, the outcome of which was counted as number of laughter events during $\mathrm{T}$, did not correlate with any activity that was evoked during I (Table 1). Hence, there was no evidence of a higher emotional arousal in the right ventral anterior insular cortex during I. In this situation, neuronal responses that are associated with effective laughter appear to be suppressed. This finding accords with evidence that the downregulation of a feeling of amusement is directly correlated with the downregulation of autonomic control (Giuliani et al., 2008). At this juncture, we point out that, with a view to minimizing movement-associated artifacts in humor-, smiling-, and ticklish-laughter-targeted fMRI studies, the participants are usually instructed to refrain from laughing during the monitoring process. This circumstance could explain why, to the best of our knowledge, activation of the right ventral anterior insula in response to an amusing situation has been observed only by us in the context of spontaneous, overt laughter.

\section{Voluntary laughter}

During $\mathrm{V}$, no signs of specific activity in the right ventral anterior insular cortex were detected (Fig. 2A). In contrast to the situation during $T$, in which the participants did not always respond to the stimulus of tickling with a spontaneous burst of laughter, during $\mathrm{V}$ the burst of laughter was produced on demand. If laughter on demand were specifically associated with activity in the right ventral anterior insular cortex, then it would have been registered. However, instead, a massive effect was bilaterally observed in the dorsal mid-/anterior portions, which greatly exceeded the activity that was recorded in these regions during T (Fig. 2B). A voluntary reproduction of one's own bodily signs of emotion involves a re-experience (embodiment) of the corresponding emotion (Niedenthal, 2007). This finding is consistent with the essence of the James-Lange theory, which postulates that emotional feelings arise from bodily sensations (Lange and James, 1967). In this sense, the heightened bilateral mid-/anterior insular 
activity could signify that the mechanical activation of the vocal apparatus involved in laughter on demand provokes the feelings that are associated with true laughter. However, other explanations are also legitimate. For instance, activation of the dorsal mid-/anterior portions of the insula is believed to imply a deliberate control of an emotional state (Wager and Barrett, 2004). To facilitate the production of laughter on demand, individuals may actively conjure up a mental picture of the tickling sensation and/or of the associated feelings or, alternatively, of another imagined humorous setup. The former explanation would account for the observed concomitant activities in the posterior sensory portion of the insula. The imagined amusing situation might, in turn, have facilitated the evocation of true laughter, as was the case in some of our participants. Hence, although $\mathrm{V}$ appears to be associated with emotional feeling, it lacks the typical characteristics of emotional arousal.

\section{CONCLUSIONS}

Imaging data on laughter-related situations are scarce and have been garnered primarily in connection with either the experience of humor (Mobbs et al., 2003; Watson et al., 2007; Schwartz et al., 2008) or passive listening to laughter (McGettigan et al., 2015). As far as we are aware, our study is the only one of its kind in which fMRI activity was recorded during the production of audible laughter. Available data indicate that spontaneous and voluntary forms of laughter rely on separate as well as on shared pathways (Scott et al., 2014). By investigating the regional activation patterns in the insular cortex during $\mathrm{T}$, I, and V, we have considered autonomic regulation as an underlying pervasive mechanism. Only laughter that accompanied real amusement was associated with activity in the right ventral anterior insular cortex, which is suggestive of immediate emotional arousal. However, our conclusions are drawn with some reservations. The reported lateralization effect was revealed as the outcome of a correlation analysis and might have been attributable not only to differences in neuronal activation but also to methodological or evaluation factors (e.g., the direction of magnetization or a lack of statistical power). Additional investigations are now called for to confirm the laughter-related regulation of emotional arousal. We would recommend a focus on the activity generated in the insular cortex and on its regional distribution therein.

\section{ACKNOWLEDGMENTS}

We thank Andrea Samson (University of Geneva) and Jörg Pfannmöller (University of Greifswald) for their appraisal of our article in manuscript form.

\section{CONFLICT OF INTEREST STATEMENT}

The authors have no conflicts of interest.

\section{ROLE OF AUTHORS}

All authors had full access to all the data in the study and take responsibility for the integrity of the data and the accuracy of the data analysis. Study concept and design: EW, BW, ML, MRC. Acquisition of data: EW, ML, KF. Analysis and interpretation of data: EW, BW, ML, KF. Drafting of the manuscript: EW. Critical revision of the manuscript for important intellectual content: BW, ML, KF, MRC. Statistical analysis: EW, BW, KF. Administrative, technical, and material support: ML. Study supervision: MRC.

\section{LITERATURE CITED}

Allen GV, Saper CB, Hurley KM, Cechetto DF. 1991. Organization of visceral and limbic connections in the insular cortex of the rat. J Comp Neurol 311:1-16.

Allman JM, Tetreault NA, Hakeem AY, Manaye KF, Semendeferi K, Erwin JM, Park S, Goubert V, Hof PR. 2010. The von Economo neurons in frontoinsular and anterior cingulate cortex in great apes and humans. Brain Struct Funct 214:495-517.

Altafullah I, Shipley C, Buchwald J. 1988. Voiced calls evoked by hypothalamic-stimulation in the cat. Exp Brain Res 71:21-32.

Anders S, Lotze M, Erb M, Grodd W, Birbaumer N. 2004. Brain activity underlying emotional valence and arousal: a response-related fMRI study. Hum Brain Mapp 23:200-209.

Butti C, Hof PR. 2010. The insular cortex: a comparative perspective. Brain Struct Funct 214:477-493.

Butti C, Santos M, Uppal N, Hof PR. 2013. Von Economo neurons: clinical and evolutionary perspectives. Cortex 49 : 312-326.

Butti C, Sherwood CC, Hakeem AV, Allman JM, Hof PR. 2009. Total number and volume of von Economo neurons in the cerebral cortex of cetaceans. J Comp Neurol 515:243259.

Carel C, Albucher JF, Manelfe C, GuiraudChaumeil B, Chollet F. 1997. Fou rire prodromique heralding a left internal carotid artery occlusion. Stroke 28:2081-2083.

Carlsson K, Petrovic P, Skare S, Petersson K, Ingvar M. 2000. Tickling expectations: Neural processing in anticipation of a sensory stimulus. J Cogn Neurosci 12:691-703.

Cechetto DF, Chen SJ. 1990. Subcortical sites mediating sympathetic responses from insular cortex in rats. Am J Physiol 258:R245-R255.

Craig A. 2009. How do you feel-now? The anterior insula and human awareness. Nat Rev Neurosci 10:59-70.

Craig AD. 2003. Interoception: the sense of the physiological condition of the body. Curr Opin Neurobiol 13:500-505.

Craig AD. 2005. Forebrain emotional asymmetry: a neuroanatomical basis? Trends in Cognitive Sciences 9:566-571.

Damasio AR, Grabowski TJ, Bechara A, Damasio H, Ponto LLB, Parvizi J, Hichwa RD. 2000. Subcortical and cortical brain activity during the feeling of self-generated emotions. Nat Neurosci 3:1049-1056.

Davis PJ, Zhang SP, Winkworth A, Bandler R. 1996. Neural control of vocalization: respiratory and emotional influences. J Voice 10:23-38.

Demaree HA, Schmeichel BJ, Robinson JL, Everhart DE. 2004. Behavioural, affective, and physiological effects of 
negative and positive emotional exaggeration. Cogn Emot 18:1079-1097.

Dujardin E, Jurgens U. 2006. Call type-specific differences in vocalization-related afferents to the periaqueductal gray of squirrel monkeys (Saimiri sciureus). Behavioural Brain Research 168:23-36.

Dupont S, Bouilleret V, Hasboun D, Semah F, Baulac M. 2003. Functional anatomy of the insula: new insights from imaging. Surg Radiol Anat 25:113-119.

Evrard HC, Forro T, Logothetis NK. 2012. von Economo neurons in the anterior insula of the macaque monkey. Neuron $74: 482-489$.

Evrard HC, Logothetis NK, Craig A. 2014. Modular architectonic organization of the insula in the macaque monkey. J Comp Neurol 522:64-97.

Giuliani NR, McRae K, Gross JJ. 2008. The up- and downregulation of amusement: experiential, behavioral, and autonomic consequences. Emotion 8:714-719.

Hakeem AY, Sherwood CC, Bonar CJ, Butti C, Hof PR, Allman JM. 2009. von Economo neurons in the elephant brain. Anat Rec Adv Integr Anat Evol Biol 292:242-248.

Hennenlotter A, Schroeder U, Erhard P, Castrop F, Haslinger B, Stoecker D, Lange KW, Ceballos-Baumann AO. 2005. A common neural basis for receptive and expressive communication of pleasant facial affect. Neuroimage 26:581-591.

Jurgens U. 2009. The neural control of vocalization in mammals: a review. J Voice 23:1-10.

Kreibig SD, Samson AC, Gross JJ. 2013. The psychophysiology of mixed emotional states. Psychophysiology 50:799-811.

Kuniecki M, Urbanik A, Sobiecka B, Kozub J, Binder M. 2003. Central control of heart rate changes during visual affective processing as revealed by fMRI. Acta Neurobiol Exp 63:39-48.

Lackner HK, Weiss EM, Hinghofer-Szalkay H, Papousek I. 2014. Cardiovascular effects of acute positive emotional arousal. Appl Psychophysiol Biofeedback 39:9-18.

Lane RD, McRae K, Reiman EM, Chen K, Ahern GL, Thayer JF. 2009. Neural correlates of heart rate variability during emotion. Neuroimage 44:213-222.

Lange CG, James W. 1967. In: Dunlop K, editor. The emotions. New York: Hafner Publishing Co.

Lauterbach EC, Cummings JL, Kuppuswamy PS. 2013. Toward a more precise, clinically-informed pathophysiology of pathological laughing and crying. Neurosci Biobehav Rev 37:1893-1916

McGettigan C, Walsh E, Jessop R, Agnew Z, Sauter D, Warren J, Scott S. 2015. Individual differences in laughter perception reveal roles for mentalizing and sensorimotor systems in the evaluation of emotional authenticity. Cereb Cortex 25:246-257.

Mesulam MM, Mufson EJ. 1982a. Insula of the Old-World monkey. 1. Architectonics in the insulo-orbito-temporal component of the paralimbic brain. J Comp Neurol 212:1-22.

Mesulam MM, Mufson EJ. 1982b. Insula of the Old-World monkey. 3. Efferent cortical output and comments on function. J Comp Neurol 212:38-52.

Meyer S, Strittmatter M, Fischer C, Georg T, Schmitz B. 2004. Lateralization in autononic dysfunction in ischemic stroke involving the insular cortex. Neuroreport 15:357-361.

Mobbs D, Greicius MD, Abdel-Azim E, Menon V, Reiss AL. 2003. Humor modulates the mesolimbic reward centers. Neuron 40:1041-1048.

Moran JM, Wig GS, Adams RB, Janata P, Kelley WM. 2004. Neural correlates of humor detection and appreciation. Neuroimage 21:1055-1060.

Mufson EJ, Mesulam MM. 1982. Insula of the Old-World monkey. 2. Afferent cortical input and comments on the claustrum. J Comp Neurol 212:23-37.
Niedenthal PM. 2007. Embodying emotion. Science 316: 1002- 1005

Nimchinsky EA, Gilissen E, Allman JM, Perl DP, Erwin JM, Hof PR. 1999. A neuronal morphologic type unique to humans and great apes. Proc Natl Acad Sci U S A 96:5268-5273.

Olausson H, Lamarre Y, Backlund H, Morin C, Wallin BG, Starck G, Ekholm S, Strigo I, Worsley K, Vallbo AB, Bushnell MC. 2002. Unmyelinated tactile afferents signal touch and project to insular cortex. Nat Neurosci 5:900-904.

Oppenheimer SM, Gelb A, Girvin JP, Hachinski VC. 1992. Cardiovascular effects of human insular cortex stimulation. Neurology 42:1727-1732.

Osseby G, Manceau E, Huet F, Becker F, Chouchane W, Durand C, Giroud M. 1999. "Fou rire prodromique" as the heralding symptom of lenticular infarction, caused by dissection of the internal carotid artery in a 12-year-old boy. Eur J Paediatr Neurol 3:133-136.

Phillips ML, Young AW, Senior C, Brammer M, Andrew C, Calder AJ, Bullmore ET, Perrett DI, Rowland D, Williams SCR, Gray JA, David AS. 1997. A specific neural substrate for perceiving facial expressions of disgust. Nature 389:495-498.

Ramachandran VS. 1998. The neurology and evolution of humor, laughter, and smiling: the false alarm theory. Med Hypoth 51:351-354.

Riecker A, Ackermann H, Wildgruber D, Dogil G, Grodd W. 2000. Opposite hemispheric lateralization effects during speaking and singing at motor cortex, insula and cerebellum. Neuroreport 11:1997-2000.

Sander K, Scheich H. 2005. Left auditory cortex and amygdala, but right insula dominance for human laughing and crying. J Cogn Neurosci 17:1519-1531.

Schwartz S, Ponz A, Poryazova R, Werth E, Boesiger P, Khatami R, Bassetti CL. 2008. Abnormal activity in hypothalamus and amygdala during humour processing in human narcolepsy with cataplexy. Brain 131:514-522.

Scott SK, Lavan N, Chen S, McGettigan C. 2014. The social life of laughter. Trends Cogn Sci 18:618-620.

Suls J. 1972. In: Goldstein J, McGhee P, editors. A two stagestage model for the appreciation of jokes and cartoons: an information processing analysis. New York: Academic Press. p 81-100.

Uddin LQ. 2015. Salience processing and insular cortical function and dysfunction. Nat Rev Neurosci 16:55-61.

von Economo C. 1926. A new type of special cells of lobus einguli and lobus insulae. Z Gesamte Neurol Psychiatrie 100:704-710.

Wager TD, Barrett LF. 2004. From affect to control: Functional specialization of the insula in motivation and regulation. Online publication at PsycExtra http://www apa org/ pubs/databases/psycextra.

Watson KK, Matthews BJ, Allman JM. 2007. Brain activation during sight gags and language-dependent humor. Cereb Cortex 17:314-324.

Wattendorf E, Westermann B, Fiedler K, Kaza E, Lotze M, Celio MR. 2013. Exploration of the neural correlates of ticklish laughter by functional magnetic resonance imaging. Cereb Cortex 23:1280-1289.

Wild B, Rodden FA, Grodd W, Ruch W. 2003. Neural correlates of laughter and humour. Brain 126:2121-2138.

Wild B, Rodden FA, Rapp A, Erb M, Grodd W, Ruch W. 2006. Humor and smiling-cortical regions selective for cognitive, affective, volitional components. Neurology 66:887-893.

Wilson SAK. 1924. Some problems in neurology: II; Pathological laughing and crying. Journal of Neurology and Psychopathology 4:299-333.

Yasui Y, Breder CD, Saper CB, Cechetto DF. 1991. Autonomic responses and efferent pathways from the insular cortex in the rat. J Comp Neurol 303:355-374. 